\title{
COMPLEMENT ACTIVATION BY STAPHYLOCOCCUS EPIDERMIDIS BIOFILM IS LESS PRONOUNCED IN NEONATES AS COMPARED TO ADULTS
}

\author{
H. Granslo ${ }^{1}$, C. Klingenberg ${ }^{1,2}$, E.A. Fredheim ${ }^{1}$, G. Acharya ${ }^{3}$, T.E. Mollnes ${ }^{4,5}$, T. Flægstad ${ }^{1,2}$ \\ ${ }^{1}$ Pediatric Research Group, Department of Clinical Medicine, University of Tromsø, ${ }^{2}$ Department of \\ Pediatrics, University Hospital of North-Norway, ${ }^{3}$ Women's Health and Perinatology Research Group, \\ Department of Clinical Medicine, University of Tromsø, Tromsø, ${ }^{4}$ Department of Immunology and \\ Transfusion Medicine, Nordland Hospital, Bodø, ${ }^{5}$ Department of Medical Biology, University of Tromsø, \\ Tromsø, Norway
}

Background: The innate immune system in neonates is immature, making them more susceptible to infections. Staphylococcus epidermidis (SE) is the most frequent cause of late-onset sepsis in neonates. SE produces a biofilm consisting of the polysaccharide intercellular adhesin (PIA), which may have a central role in the pathogenesis of these infections. There is little knowledge on how the complement, an essential part of innate immunity, reacts to SE biofilm associated infections. The aim of this study was to investigate how SE biofilm activates the complement cascade in neonates compared to adults.

Material and method: Cord blood from term born neonates $(n=7)$ and blood from healthy adults $(n=7)$ was examined for complement activation using an ex vivo whole blood model incubated with SE1457, a PIAbiofilm producing strain, and SE1457-M10, its isogenic mutant producing a non-PIA biofilm.

Results: Both SE1457 and SE1457-M10 induced a strong C1-activation, indicating that the complement cascade was activated through the classical pathway both in neonates and adults. In general, the complement activation induced by SE1457 was significantly higher than that induced by SE1457-M10 $(\mathrm{p}=0.018)$. The activation was significantly stronger in adults than in neonates both when stimulating with SE1457 ( $\mathrm{p}=$ 0.004) and SE1457-M10 ( $=0.04)$.

Conclusion: The complement cascade in this model of a $S E$ biofilm infection was induced through the classical pathway. The poorer complement response in neonates as compared to adults may explain why neonates are more susceptible to these infections. 